\title{
Las formas de tratamiento pronominales en Mateare, Managua: usos y actitudes lingüísticas
}

\section{The pronominal forms of treatment in Mateare, Managua: linguistic uses and attitudes}

(c) UNAN-Managua

Recibido: 24 de junio 2021

Aprobado: 24 de julio 2021

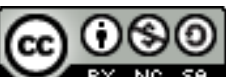

\author{
Leticia Rodríguez \\ Investigadora independiente \\ cianaguez@gmail.com \\ https://orcid.org/0000-0002-1763-171X \\ Karla Rodríguez \\ Investigadora independiente \\ karlaprodriguez970@gmail.com \\ https://orcid.org/0000-0001-5744-9974 \\ Judith Esperanza Cisnero Loáisiga \\ Investigadora independiente \\ yuyucisne2015@gmail.com \\ https://orcid.org/0000-0003-3807-6951 \\ Daniel Antonio Rodríguez Estrada \\ Investigador independiente \\ dr7077560@gmail.com \\ https://orcid.org/0002-0643-485K
}

\section{RESUMEN}

Es bien sabido que las formas de tratamiento pronominales varían según los lugares donde se emplean. Nicaragua es considerada plenamente voseante, sin embargo, el uso del vos se da de acuerdo a los parámetros simétricos y asimétricos establecidos por Brown y Gilman (1960). La presente investigación trata de las formas de tratamiento pronominales vos, tú y usted empleadas por los habitantes del barrio José Benito Escobar de Mateare, municipio del departamento de Managua, cuya finalidad es analizar dialectológicamente el tratamiento tanto en relaciones simétricas, asimétricas y de distanciamiento, también en situaciones pragmáticas. De la misma manera, se estudió el tratamiento desde la perspectiva de las actitudes lingüísticas vinculadas con estos pronombres de la segunda persona del singular. Para ello, se aplicaron encuestas a un total de 24 informantes. Entre los resultados más relevantes destacamos que la edad es determinante para decidir el tratamiento hacia el interlocutor, ya que, si este es menor que el emisor, lo más seguro es que se lo trate de vos, pero si es mayor se lo tratará de usted. Otra conclusión importante es que el usted es el tratamiento dominante cuando se acaba de conocer a una persona, pero a medida que la confianza crece, aumentan las posibilidades de un cambio de pronombres a favor de vos. Además, esta investigación corroboró que el tuteo sigue siendo prácticamente inexistente en Nicaragua, principalmente en la lengua hablada.

Palabra claves: actitud lingüística, pronominal, formas de tratamiento.

\section{SUMMARY}

It is well known that pronominal forms of treatment vary according to the places where they are used. Nicaragua is considered fully use of the first person pronoun vos, however, the use of the pronoun vos occurs according to the symmetric and asymmetric parameters established by Brown and Gilman (1960). This research deals with the forms of pronominal treatment vos, tú and usted used by the inhabitants in the neighborhood of José Benito Escobar in the municipality of Mateare, department of Managua, whose purpose is to dialectologically analyze the treatment in both symmetric, asymmetric and distancing relationships, also in pragmatic situations. In the same way, the treatment was studied from the perspective of the linguistic attitudes associated with these pronouns of the second person singular. For this, surveys were applied to a total of 24 informants. Among the most relevant results, we highlighted the age as a determining factor in deciding the treatment of the interlocutor, since if this is less than the sender, it is most likely that it will be treated as vos, but if it is older it will be treated as usted. Another important conclusion is that usted is the dominant treatment when you have just met a person, but as trust grows, the chances of a pronoun change in support of vos. Furthermore, this investigation confirmed that the use of tú continues to be practically non-existent in Nicaragua, mainly in the spoken language.

Key word: linguistic attitude, pronominal, forms of treatment. 


\section{INTRODUCCIÓN}

La sociolingüística es una de las ciencias más jóvenes que se enfoca en el estudio de las relaciones entre la lengua y la sociedad. Se trata de una vasta área de investigación que surgió como consecuencia de la interacción entre la lingüística y diversas disciplinas, entre ellas la sociología.

Así mismo, Uribe, (1970) expresa que "la sociolingüística es un término usado apenas en 1952 por Currie, para designar una disciplina que sigue paralelos de la sicolingüística, la etnolingüística, la lingüística y la sociología" (p.40). Por otro lado, la sociolingüística nace a partir del desarrollo de los estudios sobre el carácter social del lenguaje y sobre su función comunicativa, es decir que en un principio no estaba claramente diferenciada de los estudios sociológicos.

Las formas de tratamiento constituyen un tema que ha sido objeto de un sinnúmero de estudios en los países centroamericanos desde diversas perspectivas: dialectología, sociolingüística y actitudes lingüísticas, sin embargo, la mayoría de estas investigaciones se han enfocado en ciudades grandes, normalmente en cabeceras departamentales, lo cual indica que hacen falta estudios que den cuenta de cómo es el tratamiento en pueblos pequeños, en zonas semirurales y comunidades rurales para tener una idea más completa del tratamiento y las creencias y actitudes lingüísticas hacia estos pronombres. Cabe destacar que con este último enfoque son pocas las investigaciones que se han realizado en Nicaragua, pues hasta la fecha solo contamos con la tesis doctoral de Ane Christiansen (2012), quien estudió las creencias y actitudes lingüísticas hacia las formas de tratamiento en Managua.

El carácter sociolingüístico de este estudio se debe a que hemos tenido en cuenta dos variables sociales, el sexo, que se enfoca en los roles y el trabajo que socialmente se le puede asignar a hombres o a mujeres en las diferentes sociedades; y la variable edad cuyo enfoque recae en las diferencias diageneracionales que se manifiestan en el habla.

Cabe mencionar también que esta investigación sigue la teoría de Brown y Gilman (1960), citados por Amanda Castro (2001, p. 5), quienes realizaron un estudio sobre el trato pronominal en varios idiomas: inglés, francés, alemán y español. Analizaron los tipos de tratamiento: a) T-V; b) T-T; y c) V-V, en donde $T=$ tú y $V=$ usted. Los incisos b y c pertenecen "a la categoría de solidaridad recíproca" y a "a la categoría de poder no recíproco," que implica generalmente una relación de poder, dado que el empleo de las formas de tratamiento se rige bajo estos parámetros, no solo en Nicaragua, sino en muchos países hispanohablantes. 


\section{MATERIAL Y MÉTODOS}

\section{Tipo de investigación}

Esta investigación es de tipo descriptiva mixta, es decir cualitativa y cuantitativa, ya que estudia el empleo de las formas de tratamiento pronominales vos, tú y usted en los hablantes de Mateare y analiza algunas actitudes lingüísticas hacia estos pronombres.

\section{Universo}

El universo de la investigación lo conforman la totalidad de hispanohablantes que habitan en el barrio José Benito Escobar de Mateare.

\section{Muestra}

La muestra está basada en veinticuatro hispanohablantes, habitantes del Bo. José Benito Escobar, del municipio de Mateare, en el departamento de Managua. Dicha muestra está subdividida en doce hombres y doce mujeres, de los cuales seis serán personas mayores, de 55 años a más y seis, personas jóvenes de 18 a 45 años, respectivamente.

\section{Los informantes}

Los informantes fueron elegidos a partir de las necesidades básicas del estudio. En este caso se trabajó con personas con un nivel de educación básico, bachiller como máximo, que no hayan sido influenciados en su habla por los estándares educativos universitarios. La elección de las edades estuvo adecuada para realizar una comparación entre las formas de tratamiento de los hablantes jóvenes y aquellos considerados mayores; y entre los hablantes del género femenino y los del masculino.

Figura 1. Niveles de instrucción de los informantes

\section{Nivel de instrucción de los informantes}

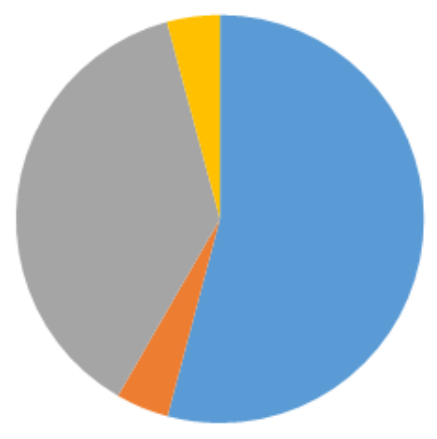

- Primaria completa (13)

- Secundaria incompleta (1)

- Secundaria completa (9)

- Iletrados (1) 
De manera que lo interesante de la muestra presentada en el gráfico anterior es que todos los informantes poseen niveles básicos de instrucción formal, ya que ninguno ingresó al subsistema educativo superior, destacándose así los niveles nivel de educación de primaria completa y secundaria completa, entre los informantes.

\section{El cuestionario}

El cuestionario que se utilizará en la investigación fue adaptado del trabajo de realizado por los miembros del equipo PRESEEA- BOGOTÁ, Colombia (Proyecto para el estudio sociolingüístico del Español de España y de América), con la colaboración de Isabel Molina Martos (PRESEEA- ALCALÁ- ES). Dicho cuestionario a la vez se unificó con el cuestionario propuesto por Alemán, Picado y Talavera (2017), con el objeto de enriquecer la información requerida, según las necesidades planteadas en los objetivos de la investigación. La guía cuenta con una serie de preguntas acerca del empleo pronominal, además de otras interrogantes que se refieren al uso de estas formas de tratamiento, teniendo en cuenta las actitudes lingüísticas de los hablantes.

\section{La localidad}

El presente trabajo fue realizado en el barrio José Benito Escobar, municipio de Mateare, departamento de Managua. Este municipio fue considerado para nuestro estudio por su gran valor histórico, debido a su antigüedad. Además, con esta investigación se pretende aportar en el conocimiento del uso de las formas de tratamiento en pueblos pequeños de nuestro país.

\section{Métodos empleados}

Para el desarrollo de este estudio se aplicaron los siguientes métodos:

El método sociolingüístico, ya que el estudio compara las formas de tratamiento, teniendo en cuenta las variables sociales edad y sexo; a la vez que se estudian las actitudes lingüísticas hacia estos usos.

El método de análisis morfológico, porque el estudio se enfoca en las formas de tratamiento que pertenecen a la categoría gramatical del pronombre y explora, de manera sistemática, los posibles resultados de la investigación.

El método de análisis y síntesis se refiere a dos actividades complementarias en el estudio de relaciones complejas, lo que nos permite conocer más profundamente las realidades con las que nos enfrentamos, simplificar su descripción y construir nuevos conocimientos a partir de otros ya conocidos.

\section{RESULTADOS}

De acuerdo con los resultados de las encuestas realizadas en el municipio de Mateare, en cuanto a las formas de tratamiento que los hijos utilizan para dirigirse hacia sus madres predominó en un 100\% el ustedeo. Este 
uso recurrente indica el respeto con que los hijos tratan a las madres, parte importante de la buena educación que reciben en el hogar, como es característico en una sociedad que inculca de esta manera una forma de mostrar respeto hacia sus padres.

Tabla 1.

Tratamiento de hijos a madres y padres

\begin{tabular}{|c|c|c|c|c|c|c|c|c|c|c|}
\hline \multirow[b]{2}{*}{ Variantes } & \multicolumn{2}{|c|}{ Nivel generacional } & \multicolumn{2}{|c|}{ Nivel sexual } & \multicolumn{4}{|c|}{ Grupo individual } & \multirow[t]{2}{*}{ total } & \multirow[t]{2}{*}{$\%$} \\
\hline & Mayores & Jóvenes & Hombres & Mujeres & $\mathrm{HM}$ & $\mathrm{HJ}$ & $\overline{\mathrm{MM}}$ & $\overline{M J}$ & & \\
\hline \multicolumn{11}{|c|}{ A madres } \\
\hline Vos & 0 & 0 & 0 & 0 & 0 & 0 & 0 & 0 & 0 & $0 \%$ \\
\hline Usted & 12 & 12 & 12 & 12 & 6 & 6 & 6 & 6 & 24 & $100 \%$ \\
\hline Total & 12 & 12 & 12 & 12 & 6 & 6 & 6 & 6 & 24 & $100 \%$ \\
\hline \multicolumn{11}{|c|}{ A padres } \\
\hline Vos & 0 & 1 & 1 & 0 & 0 & 1 & 0 & 0 & 1 & $4.2 \%$ \\
\hline Usted & 12 & 11 & 11 & 12 & 6 & 5 & 6 & 6 & 23 & $95.8 \%$ \\
\hline Total & 12 & 12 & 12 & 12 & 6 & 6 & 6 & 6 & 24 & $100 \%$ \\
\hline
\end{tabular}

Por otra parte, y no lejos del dato anterior, los resultados del tratamiento hacia los padres indica que el 95.8\% usa el ustedeo, aunque cabe señalar que 4.2\% afirmó utilizar el voseo, este resultado está representado por un hombre joven quien aseguró emplear el vos por la confianza que siente hacia su padre.

Es evidente la preferencia del ustedeo en el tratamiento hacia los padres, puesto que hay consenso tanto a nivel diasexual como diageneracional. No obstante, pese a la poca utilización del voseo en esta situación, en la actualidad se está introduciendo levemente el uso de este tratamiento, lo que podría ir aumentando paulatinamente sobre todo entre los jóvenes, como está ocurriendo en áreas más urbanizadas.

Tabla 2.

Tratamiento entre hermanos

\begin{tabular}{|c|c|c|c|c|c|c|c|c|c|c|}
\hline \multirow[t]{2}{*}{ Variantes } & \multicolumn{2}{|c|}{ Nivel generacional } & \multicolumn{2}{|c|}{ Nivel sexual } & \multicolumn{4}{|c|}{ Grupo individual } & \multirow[t]{2}{*}{ total } & \multirow[t]{2}{*}{$\%$} \\
\hline & Mayores & Jóvenes & Hombres & Mujeres & $\mathrm{HM}$ & $\mathrm{HJ}$ & $\begin{array}{l}\mathrm{M} \\
\mathrm{M}\end{array}$ & $\mathrm{MJ}$ & & \\
\hline \multicolumn{11}{|c|}{ A hermanos } \\
\hline Vos & 11 & 11 & 12 & 10 & 6 & 6 & 5 & 5 & 22 & $91.7 \%$ \\
\hline Usted & 1 & 1 & 0 & 2 & 0 & 0 & 1 & 1 & 2 & $8.3 \%$ \\
\hline Total & 12 & 12 & 12 & 12 & 6 & 6 & 6 & 6 & 24 & $100 \%$ \\
\hline \multicolumn{11}{|c|}{ A hermanas } \\
\hline Vos & 11 & 12 & 12 & 11 & 6 & 6 & 5 & 6 & 23 & $95.8 \%$ \\
\hline Usted & 1 & 0 & 0 & 1 & 0 & 0 & 1 & 0 & 1 & $4.2 \%$ \\
\hline Total & 12 & 12 & 12 & 12 & 6 & 6 & 6 & 6 & 24 & $100 \%$ \\
\hline
\end{tabular}


En cuanto a la forma de tratamiento entre hermanos, encontramos las siguientes variaciones: el 91.7\% trata a sus hermanos de vos, pero un 8.3\% los trata de usted, este último porcentaje está representado por mujeres, una joven y una mayor, lo que resulta interesante en esta forma de tratamiento es que se utiliza para dirigirse a sus hermanos varones mayores. Acerca del trato hacia las hermanas, la mayoría usa el vos (95.8\%) solamente una mujer mayor admitió dirigirse a su hermana con el usted, representado por un $4.2 \%$.

Cabe recalcar que, en cuanto al trato entre hermanos, todos los varones afirmaron usar el voseo y, aunque la mayoría de mujeres también lo utilizaron, dos de ellas refirieron el uso del ustedeo hacia sus hermanos mayores, lo cual indica un cierto nivel de educación sobre el respeto a las personas de mayor edad.

Tabla 3.

Tratamiento entre parejas

\begin{tabular}{|c|c|c|c|c|c|c|c|c|c|c|}
\hline Variantes & \multicolumn{2}{|c|}{ Nivel generacional } & \multicolumn{2}{|c|}{ Nivel sexual } & \multicolumn{3}{|c|}{ Grupo individual } & total & \multirow{2}{*}{$\%$} \\
\cline { 2 - 10 } & Mayores & Jóvenes & Hombres & Mujeres & $\mathrm{HM}$ & $\mathrm{HJ}$ & $\begin{array}{c}\mathrm{M} \\
\mathrm{M}\end{array}$ & $\mathrm{MJ}$ & & \\
\hline Vos & 12 & 12 & 12 & 12 & 6 & 6 & 6 & 6 & 24 & $100 \%$ \\
\hline Usted & 0 & 0 & 0 & 0 & 0 & 0 & 0 & 0 & 0 & $0 \%$ \\
\hline Total & 12 & 12 & 12 & 12 & 6 & 6 & 6 & 6 & 24 & $100 \%$ \\
\hline
\end{tabular}

La forma de tratamiento entre parejas en Mateare resultó ser muy igualitaria, puesto que el 100\% de los entrevistados aseguran utilizar el voseo al comunicarse con sus parejas sentimentales. Este resultado infiere una respuesta positiva en cuanto a la igualdad de género, lo que indica relaciones de carácter horizontal, al menos en el tratamiento.

Tabla 4.

Tratamiento de nietos a abuelos

\begin{tabular}{|c|c|c|c|c|c|c|c|c|c|c|}
\hline \multirow[t]{2}{*}{ Variantes } & \multicolumn{2}{|c|}{ Nivel generacional } & \multicolumn{2}{|c|}{ Nivel sexual } & \multicolumn{4}{|c|}{ Grupo individual } & \multirow[t]{2}{*}{ total } & \multirow[t]{2}{*}{$\%$} \\
\hline & Mayores & Jóvenes & Hombres & Mujeres & $\mathrm{HM}$ & $\mathrm{HJ}$ & $\begin{array}{l}\mathrm{M} \\
\mathrm{M}\end{array}$ & $\overline{M J}$ & & \\
\hline \multicolumn{11}{|c|}{ A abuelos } \\
\hline Vos & 0 & 0 & 0 & 0 & 0 & 0 & 0 & 0 & 0 & $0 \%$ \\
\hline Usted & 12 & 12 & 12 & 12 & 6 & 6 & 6 & 6 & 24 & $100 \%$ \\
\hline Total & 12 & 12 & 12 & 12 & 6 & 6 & 6 & 6 & 24 & $100 \%$ \\
\hline \multicolumn{11}{|c|}{ A abuelas } \\
\hline Vos & 0 & 0 & 0 & $\overline{0}$ & $\overline{0}$ & 0 & 0 & 0 & $\overline{0}$ & $0 \%$ \\
\hline Usted & 12 & 12 & 12 & 12 & 6 & 6 & 6 & 6 & 24 & $100 \%$ \\
\hline Total & 12 & 12 & 12 & 12 & 6 & 6 & 6 & 6 & 24 & $100 \%$ \\
\hline
\end{tabular}


Al contrario de la situación anterior, el resultado del trato de nietos hacia abuelos fue un 100\% de ustedeo. De manera que esta demostración de respeto hacia los adultos mayores de la familia fue igual entre hombres y mujeres, tanto en los mayores como los de menor edad.

En este caso, el consenso obtenido en cuanto al uso de usted, demuestra una vez más que este pronombre sigue representando el tratamiento de respeto por excelencia.

Tabla 5.

Tratamiento entre amigos

\begin{tabular}{|c|c|c|c|c|c|c|c|c|c|c|}
\hline \multirow[t]{2}{*}{ Variantes } & \multicolumn{2}{|c|}{ Nivel generacional } & \multicolumn{2}{|c|}{ Nivel sexual } & \multicolumn{4}{|c|}{ Grupo individual } & \multirow[t]{2}{*}{ total } & \multirow[t]{2}{*}{$\%$} \\
\hline & Mayores & Jóvenes & Hombres & Mujeres & $\overline{\mathrm{HM}}$ & $\mathrm{HJ}$ & $\begin{array}{l}\mathrm{M} \\
\mathrm{M}\end{array}$ & $\overline{M J}$ & & \\
\hline \multicolumn{11}{|c|}{ A amigo } \\
\hline Vos & 9 & 11 & 10 & 10 & 5 & 5 & 4 & 6 & 20 & $83.3 \%$ \\
\hline Usted & 3 & 0 & 1 & 2 & 1 & 0 & 2 & 0 & 3 & $12.5 \%$ \\
\hline Vos usted & 0 & 1 & 1 & 0 & 0 & 1 & 0 & 0 & 1 & $4.2 \%$ \\
\hline Total & 12 & 12 & 12 & 12 & 6 & 6 & 6 & 6 & 6 & $100 \%$ \\
\hline \multicolumn{11}{|c|}{ A amiga } \\
\hline Vos & 9 & 11 & 10 & 10 & 5 & 5 & 4 & 6 & 20 & $83.3 \%$ \\
\hline Usted & 3 & 0 & 1 & 2 & 1 & 0 & 2 & 0 & 3 & $12.5 \%$ \\
\hline Vos usted & 0 & 1 & 1 & 0 & 0 & 1 & 0 & 0 & 1 & $4.2 \%$ \\
\hline Total & 12 & 12 & 12 & 12 & 6 & 6 & 6 & 6 & 24 & $100 \%$ \\
\hline
\end{tabular}

Sobre el trato hacia el amigo, el porcentaje para el uso del voseo fue de $83.3 \%$, este uso se presentó con más concurrencia en mujeres jóvenes entre las cuales las seis participantes señalaron dirigirse de vos a sus amigos. Para el ustedeo hubo un porcentaje de 12.5\%, cabe destacar que dicho uso se presentó solamente entre personas mayores, un hombre y dos mujeres.

Al analizar la forma de tratamiento hacia los amigos los resultados son bastante parecidos a los anteriores, con un $83.3 \%$ en el uso del voseo y $12.5 \%$ para el ustedeo; nuevamente fueron tres personas mayores las que refirieron este último uso.

El último 4.2\% está representado por un informante joven quien expresó utilizar el ustedeo y el voseo según la edad de los amigos o amigas con quienes se comunica. Este participante señaló que a las personas de mayor edad las trata de usted por el respeto que se merecen y con los jóvenes de su edad, o menores que él, usa el vos, porque ameritan una relación de más confianza. Este fenómeno se repite en los siguientes cuestionamientos, en el tratamiento de este participante joven se encuentra entre el voseo y el ustedeo en dependen- 
cia de la edad de las personas con quienes conversa.

Tabla 6.

Tratamiento a desconocidos

\begin{tabular}{|c|c|c|c|c|c|c|c|c|c|c|}
\hline \multirow[t]{2}{*}{ Variantes } & \multicolumn{2}{|c|}{ Nivel generacional } & \multicolumn{2}{|c|}{ Nivel sexual } & \multicolumn{4}{|c|}{ Grupo individual } & \multirow[t]{2}{*}{ Total } & \multirow[t]{2}{*}{$\%$} \\
\hline & Mayores & Jóvenes & Hombres & Mujeres & $\mathrm{HM}$ & $\mathrm{HJ}$ & $\mathrm{MM}$ & $\overline{\mathrm{MJ}}$ & & \\
\hline \multicolumn{11}{|c|}{ Al desconocido } \\
\hline Vos & 8 & 2 & 5 & 5 & 4 & 1 & 4 & 1 & 10 & $41.7 \%$ \\
\hline Usted & 4 & 9 & 6 & 7 & 2 & 4 & 2 & 5 & 13 & $54.2 \%$ \\
\hline $\begin{array}{l}\text { Vos us- } \\
\text { ted }\end{array}$ & 0 & 1 & 1 & 0 & 0 & 1 & 0 & 0 & 1 & $4.2 \%$ \\
\hline Total & 12 & 12 & 12 & 12 & 6 & 6 & 6 & 6 & 24 & $100 \%$ \\
\hline \multicolumn{11}{|c|}{ A la desconocida } \\
\hline Vos & 8 & 2 & 5 & 5 & 4 & 1 & 4 & 1 & 10 & $41.7 \%$ \\
\hline Usted & 4 & 9 & 6 & 7 & 2 & 4 & 2 & 5 & 13 & $54.2 \%$ \\
\hline $\begin{array}{c}\text { Vos } \sim \text { us- } \\
\text { ted }\end{array}$ & 0 & 1 & 1 & 0 & 0 & 1 & 0 & 0 & 1 & $4.2 \%$ \\
\hline Total & 12 & 12 & 12 & 12 & 6 & 6 & 6 & 6 & 6 & $100 \%$ \\
\hline
\end{tabular}

Referente al trato hacia los desconocidos, los encuestados indicaron utilizar el voseo en un $41.7 \%$ tanto a desconocidos, como a desconocidas. El 54.2\% usó el ustedeo. Cabe destacar que este es el primer caso en el que no se observa consenso por parte de los informantes, lo cual se debe a que el factor edad es determinante para elegir el tratamiento que dispensará al interlocutor, pues si este es más joven que el emisor, se lo tratará de vos, pero si es una persona mayor recibirá un usted de respeto y distanciamiento.

Tabla 7.

Tratamiento hacia maestros

\begin{tabular}{|c|c|c|c|c|c|c|c|c|c|c|c|c|}
\hline Variantes & Nivel generacional & \multicolumn{2}{|c|}{ Nivel sexual } & \multicolumn{3}{|c|}{ Grupo individual } & To- & $\%$ \\
& Mayores & Jóvenes & Hombres & Mujeres & $\mathrm{HM}$ & $\mathrm{HJ}$ & $\begin{array}{c}\mathrm{M} \\
\mathrm{M}\end{array}$ & $\mathrm{MJ}$ & \\
\hline \multicolumn{8}{|c|}{ Al maestro } \\
\hline Vos & 0 & 0 & 0 & 0 & 0 & 0 & 0 & 0 & 0 & 0 \\
\hline Usted & 12 & 12 & 12 & 12 & 6 & 6 & 6 & 6 & 24 & $100 \%$ \\
\hline Total & 12 & 12 & 12 & 12 & 6 & 6 & 6 & 6 & 24 & $100 \%$ \\
\hline \multicolumn{8}{|c|}{ A la maestra } \\
\hline Vos & 0 & 0 & 0 & 1 & 0 & 0 & 0 & 1 & 1 & $4.2 \%$ \\
\hline Usted & 12 & 12 & 12 & 11 & 6 & 6 & 6 & 5 & 23 & $95.8 \%$ \\
\hline Total & 12 & 12 & 12 & 12 & 6 & 6 & 6 & 6 & 24 & $100 \%$ \\
\hline
\end{tabular}


El resultado anterior coincide con las diferencias encontradas a nivel diageneracional, pues la mayoría de los que utilizan el voseo son los encuestados mayores, o sea, ocho de los doce participantes; por el contrario, los encuestados más jóvenes usan el ustedeo para tratar a los desconocidos. Cabe mencionar que en cuanto a la variable sexo, no se observan diferencias significativas.

En el tratamiento hacia los maestros prevalece el uso del ustedeo, con la única variación de una mujer joven que refirió tratar de vos a su maestra, representando el 4.2\%. Esta demostración de respeto al emplear el pronombre usted para dirigirse hacia los maestros ha prevalecido a lo largo del tiempo en la sociedad nicaragüense, tanto de parte de los estudiantes a los maestros, como de los padres de familia hacia los docentes, por tratarse de relaciones que implican distanciamiento.

Tabla 8.

Tratamiento hacia el jefe

\begin{tabular}{|c|c|c|c|c|c|c|c|c|c|c|}
\hline \multirow{2}{*}{ Variantes } & \multicolumn{2}{|c|}{ Nivel generacional } & \multicolumn{2}{|c|}{ Nivel sexual } & \multicolumn{3}{|c|}{ Grupo individual } & \multirow{2}{*}{ total } & $\%$ \\
\cline { 2 - 11 } & Mayores & Jóvenes & Hombres & Mujeres & $\mathrm{HM}$ & $\mathrm{HJ}$ & $\begin{array}{c}\mathrm{M} \\
\mathrm{M}\end{array}$ & $\mathrm{MJ}$ & & \\
\hline Vos & 0 & 0 & 0 & 0 & 0 & 0 & 0 & 0 & 0 & $0 \%$ \\
\hline Usted & 12 & 12 & 12 & 12 & 6 & 6 & 6 & 6 & 24 & $100 \%$ \\
\hline Total & 12 & 12 & 12 & 12 & 6 & 6 & 6 & 6 & 24 & $100 \%$ \\
\hline
\end{tabular}

La forma de tratamiento utilizada para dirigirse a los jefes fue usted, empleada por todos los informantes, tanto hombres, como mujeres. Cabe señalar que esta relación interpersonal representa relaciones de distanciamiento en las que, tanto emisor como receptor no se involucran de manera personal entre ambos, generalmente; de manera que en este tratamiento prevalece también la cortesía. También, podemos mencionar que este tipo de relaciones están sujetas a una serie de requerimientos preestablecidos, como la ética profesional.

Tabla 9.

Tratamiento hacia la asistente del hogar

\begin{tabular}{|c|c|c|c|c|c|c|c|c|c|c|}
\hline Variantes & \multicolumn{2}{|c|}{ Nivel generacional } & \multicolumn{2}{|c|}{ Nivel sexual } & \multicolumn{3}{|c|}{ Grupo individual } & To- & $\%$ \\
\cline { 2 - 11 } & Mayores & Jóvenes & Hombres & Mujeres & $\mathrm{HM}$ & $\mathrm{HJ}$ & $\begin{array}{c}\mathrm{M} \\
\mathrm{M}\end{array}$ & $\mathrm{MJ}$ & & \\
\hline Vos & 5 & 7 & 6 & 6 & 2 & 4 & 3 & 3 & 12 & $50 \%$ \\
\hline Usted & 7 & 4 & 5 & 6 & 4 & 1 & 3 & 3 & 11 & $45.8 \%$ \\
\hline $\begin{array}{c}\text { Vos us- } \\
\text { ted }\end{array}$ & 0 & 1 & 1 & 0 & 0 & 1 & 0 & 0 & 1 & $4.2 \%$ \\
\hline Total & 12 & 12 & 12 & 12 & 6 & 6 & 6 & 6 & 24 & $100 \%$ \\
\hline
\end{tabular}


En cuanto a este tratamiento, se obtuvieron los siguientes resultados: el 50\% de los entrevistados tratan a la asistente del hogar de vos y el $46.8 \%$ usa el usted. Cabe señalar que a nivel diageneracional, siete personas mayores prefirieron el uso del pronombre usted y solamente cuatro jóvenes indicaron este uso, lo cual podría ser indicio de que las personas mayores establecen más relaciones de distanciamiento con la trabajadora del hogar. Por otro lado, estos resultados refieren que el trato hacia estas trabajadoras está en dependencia de la cercanía en las relaciones sociales que se establecen con las mismas.

\section{Tabla 10.}

Tratamiento hacia el conductor del bus

\begin{tabular}{|c|c|c|c|c|c|c|c|c|c|c|}
\hline \multirow{2}{*}{ Variantes } & \multicolumn{2}{|c|}{ Nivel generacional } & \multicolumn{2}{|c|}{ Nivel sexual } & \multicolumn{3}{|c|}{ Grupo individual } & total & \multirow{2}{*}{$\%$} \\
\cline { 2 - 10 } & $\begin{array}{c}\text { Mayo- } \\
\text { res }\end{array}$ & Jóvenes & Hombres & Mujeres & HM & HJ & MM & MJ & & \\
\hline Vos & 6 & 3 & 3 & 6 & 2 & 1 & 2 & 2 & 7 & $29.2 \%$ \\
\hline Usted & 6 & 8 & 8 & 6 & 4 & 4 & 4 & 4 & 16 & $66.7 \%$ \\
\hline Vos $\sim$ usted & 0 & 1 & 1 & 0 & 0 & 1 & 0 & 0 & 1 & $4.2 \%$ \\
\hline Total & 12 & 12 & 12 & 12 & 6 & 6 & 6 & 6 & 24 & $100 \%$ \\
\hline
\end{tabular}

En el trato hacia el conductor del bus, el 66.7\% de entrevistados indicaron utilizar el ustedeo; el 29.2\% usa el voseo. En este caso prevaleció el uso del pronombre usted por el factor de distanciamiento entre los interlocutores. A nivel sociolingüístico, en el plano generacional, se observa que en los jóvenes hay consenso para emplear el usted, en cambio los mayores alternan entre vos y usted, de la misma manera, los hombres emplearon más el usted que las mujeres. Nuevamente, uno de los participantes masculinos hizo la diferencia al seleccionar el pronombre en dependencia de la edad de su interlocutor, pues si es mayor usa usted, pero si se trata de una persona menor, prefiere el voseo.

Tabla 11.

¿Trata de manera diferente a las personas por su edad?

\begin{tabular}{|c|c|c|c|c|c|c|c|c|c|c|}
\hline Variantes & \multicolumn{2}{|c|}{ Nivel generacional } & \multicolumn{2}{|c|}{ Nivel sexual } & \multicolumn{3}{|c|}{ Grupo individual } & $\begin{array}{c}\text { To- } \\
\text { tal }\end{array}$ & \\
\cline { 2 - 11 } & Mayores & Jóvenes & Hombres & Mujeres & $\mathrm{HM}$ & $\mathrm{HJ}$ & $\begin{array}{c}\mathrm{M} \\
\mathrm{M}\end{array}$ & $\mathrm{MJ}$ & & \\
\hline $\mathrm{Si}$ & 11 & 10 & 12 & 9 & 5 & 6 & 5 & 4 & 20 & $83.3 \%$ \\
\hline No & 1 & 2 & 0 & 3 & 1 & 0 & 1 & 2 & 4 & $16.6 \%$ \\
\hline Total & 12 & 12 & 12 & 12 & 6 & 6 & 6 & 6 & 24 & $100 \%$ \\
\hline
\end{tabular}

El 83.3\% indico que "si" hacen diferencias en su forma de tratar a las personas según su edad, confirmando así que este es un factor determinante en cuanto al uso de las formas de tratamiento. Por otro lado, solo el 
16.6\% afirmo "no" hacer diferencias por la edad. Cabe mencionar que en cuanto la variable social edad, se observa consenso en los informantes, ya que la mayoría está de acuerdo en que la forma de tratamiento sí varía en dependencia de la edad del interlocutor; con respecto al nivel diasexual, se observó que en los hombres hay más consenso con respecto a la importancia de la edad del interlocutor para elegir el tratamiento.

Tabla 12.

¿Cómo le gusta que le hablen?

\begin{tabular}{|c|c|c|c|c|c|c|c|c|c|c|}
\hline Variantes & \multicolumn{2}{|c|}{ Nivel generacional } & \multicolumn{2}{|c|}{ Nivel sexual } & \multicolumn{3}{|c|}{ Grupo individual } & $\begin{array}{c}\text { To- } \\
\text { tal }\end{array}$ & \\
\cline { 2 - 11 } & Mayores & jóvenes & Hombres & Mujeres & HM & HJ & $\begin{array}{c}\text { M } \\
\text { M }\end{array}$ & MJ & & \\
\hline Vos & 1 & 6 & 4 & 3 & 1 & 3 & 0 & 3 & 7 & $29.2 \%$ \\
\hline Usted & 11 & 6 & 8 & 9 & 5 & 3 & 6 & 3 & 17 & $70.8 \%$ \\
\hline Total & 12 & 12 & 12 & 12 & 6 & 6 & 6 & 6 & 24 & $100 \%$ \\
\hline
\end{tabular}

Según los datos observados en la tabla 12, los informantes prefieren ser tratados de usted (70.8\%). La mayor parte de los que eligen esta forma de tratamiento son personas mayores, como era de esperar. Solo el 29.2\% indicó la preferencia por la forma de tratamiento vos, siendo los jóvenes los que más refirieron el agrado por ser tratados con ese pronombre. Este es otro resultado que confirma que la edad del interlocutor es determinante para elegir la forma de tratamiento, ya que los mayores se sienten cómodos al ser tratados de usted como señal de respeto, mientras que el 50\% de la generación seleccionó el voseo, que este caso es indicio de juventud.

Tabla 13.

¿Cuál cree usted que es la forma correcta de tratamiento?

\begin{tabular}{|c|c|c|c|c|c|c|c|c|c|c|}
\hline Variantes & \multicolumn{2}{|c|}{ Nivel generacional } & \multicolumn{2}{|c|}{ Nivel sexual } & \multicolumn{3}{|c|}{ Grupo individual } & total & \multirow{2}{*}{$\%$} \\
\cline { 2 - 10 } & Mayores & jóvenes & Hombres & Mujeres & $\mathrm{HM}$ & $\mathrm{HJ}$ & $\begin{array}{c}\mathrm{M} \\
\mathrm{M}\end{array}$ & $\mathrm{MJ}$ & & \\
\hline Vos & 0 & 0 & 0 & 0 & 0 & 0 & 0 & 0 & 0 & $0 \%$ \\
\hline Usted & 12 & 12 & 12 & 12 & 6 & 6 & 6 & 6 & 24 & $100 \%$ \\
\hline Total & 12 & 12 & 12 & 12 & 6 & 6 & 6 & 6 & 24 & $100 \%$ \\
\hline
\end{tabular}

Aunque el uso del voseo es predominante entre las formas de tratamiento utilizadas por nuestros participantes, el 100\% de ellos refirieron opinar que la forma correcta de tratamiento es el ustedeo. Se puede considerar que los informantes eligieron usted como forma "correcta" por ser el tratamiento de respeto por excelencia, al no haber ningún otro pronombre que exprese esta connotación. 
Tabla 14.

Principales motivos de uso de cada tratamiento pronominal

\begin{tabular}{|c|c|c|c|c|c|c|c|c|c|c|}
\hline \multirow[t]{2}{*}{ Variantes } & \multicolumn{2}{|c|}{ Nivel generacional } & \multicolumn{2}{|c|}{ Nivel sexual } & \multicolumn{4}{|c|}{ Grupo individual } & \multirow{2}{*}{$\begin{array}{l}\text { To- } \\
\text { tal }\end{array}$} & \multirow[t]{2}{*}{$\%$} \\
\hline & Mayores & jóvenes & Hombres & Mujeres & $\mathrm{HM}$ & $\mathrm{HJ}$ & $\begin{array}{l}M \\
M\end{array}$ & MJ & & \\
\hline \multicolumn{11}{|c|}{ Usted } \\
\hline Respeto & 12 & 12 & 12 & 12 & 6 & 6 & 6 & 6 & 24 & $100 \%$ \\
\hline Total & 12 & 12 & 12 & 12 & 6 & 6 & 6 & 6 & 24 & $100 \%$ \\
\hline \multicolumn{11}{|c|}{ Vos } \\
\hline Respeto & 2 & 0 & 1 & 1 & 1 & 0 & 1 & 0 & 2 & $8.3 \%$ \\
\hline Edad & 1 & 0 & 0 & 1 & 0 & 0 & 1 & 0 & 1 & $4.2 \%$ \\
\hline Confianza & 9 & 12 & 11 & 10 & 5 & 6 & 4 & 6 & 21 & $87.5 \%$ \\
\hline Total & 12 & 12 & 12 & 12 & 6 & 6 & 6 & 6 & 24 & $100 \%$ \\
\hline \multicolumn{11}{|c|}{ Tú } \\
\hline Respeto & 3 & 1 & 2 & 2 & 2 & 0 & 1 & 1 & 4 & $16.6 \%$ \\
\hline Confianza & 0 & 2 & 0 & 2 & 0 & 0 & 0 & 2 & 2 & $8.3 \%$ \\
\hline Cortesía & 0 & 1 & 1 & 0 & 0 & 1 & 0 & 0 & 1 & $4.2 \%$ \\
\hline Ninguno & 9 & 8 & 9 & 8 & 4 & 5 & 5 & 3 & 17 & $70.9 \%$ \\
\hline Total & 12 & 12 & 12 & 12 & 6 & 6 & 6 & 6 & 24 & $100 \%$ \\
\hline
\end{tabular}

Según los resultados de la tabla número 14, para el uso del ustedeo el factor determinante, según los encuestados, es el respeto con el 100\% de opiniones a favor. De manera que en este estudio seguimos confirmando que la forma de tratamiento de respeto por excelencia es el ustedeo, concatenado con los resultados del ítem anterior. Con respecto al tuteo, la gran mayoría de los informantes respondieron con "ninguno", lo cual confirma que el tuteo es prácticamente inexistente en este municipio y en Nicaragua, en general. Otros aspectos por los cuales los informantes indicaron usar el pronombre tú, fueron: respeto, 16.6\%; confianza, 8.3\% y cortesía, $4.2 \%$.

En cuanto a la utilización del voseo prevaleció el factor Confianza entre los hablantes (87.5\%). Además, se consideraron elementos como: el Respeto $8.3 \%$ y la Edad $4.2 \%$. Con estos resultados se confirma, en boca de los informantes, que mientras el "usted" significa respeto, el pronombre "vos" implica confianza.

Tabla 15.

¿Cómo te dirigís a una persona que acabas de conocer?

\begin{tabular}{|c|c|c|c|c|c|c|c|c|c|c|}
\hline Variantes & \multicolumn{2}{|c|}{ Nivel generacional } & \multicolumn{2}{|c|}{ Nivel sexual } & \multicolumn{3}{|c|}{ Grupo individual } & $\begin{array}{c}\text { To- } \\
\text { tal }\end{array}$ & \multirow{2}{*}{} \\
\cline { 2 - 11 } & Mayores & jóvenes & Hombres & Mujeres & $\mathrm{HM}$ & $\mathrm{HJ}$ & $\begin{array}{c}\mathrm{M} \\
\mathrm{M}\end{array}$ & $\mathrm{MJ}$ & & \\
\hline Vos & 1 & 1 & 2 & 0 & 1 & 1 & 0 & 0 & 2 & $8.4 \%$ \\
\hline Usted & 11 & 11 & 10 & 12 & 5 & 5 & 6 & 6 & 22 & 91.6 \\
\hline Total & 12 & 12 & 12 & 12 & 6 & 6 & 6 & 6 & 24 & $100 \%$ \\
\hline
\end{tabular}


Esta investigación demostró que el $91.6 \%$ de los participantes se dirigen a alguien que acaban de conocer con el ustedeo y un $8.4 \%$ con el voseo. Estos datos muestran la falta de confianza que se manifiesta al dirigirse a una persona que recién se conoce, es por ello que se recurre al usted para imponer el distanciamiento con el interlocutor. Otro señalamiento que resulta interesante es que, a nivel diasexual, ninguna de las mujeres refirió utilizar el voseo, fueron dos varones quienes lo escogieron, demostrando que las mujeres son más cuidadosas ante este tipo de situación pragmática.

\section{Tabla 16.}

Cuando ya tenés confianza con esa persona que acabás de conocer, ¿cómo la tratás?

\begin{tabular}{|c|c|c|c|c|c|c|c|c|c|c|}
\hline Variantes & \multicolumn{2}{|c|}{ Nivel generacional } & \multicolumn{2}{|c|}{ Nivel sexual } & \multicolumn{3}{|c|}{ Grupo individual } & $\begin{array}{c}\text { To- } \\
\text { tal }\end{array}$ & \\
\cline { 2 - 11 } & Mayores & Jóvenes & Hombres & Mujeres & $\mathrm{HM}$ & $\mathrm{HJ}$ & $\begin{array}{c}\mathrm{M} \\
\mathrm{M}\end{array}$ & $\mathrm{MJ}$ & & \\
\hline Vos & 6 & 8 & 9 & 5 & 4 & 5 & 2 & 3 & 14 & $58.3 \%$ \\
\hline Usted & 6 & 4 & 3 & 7 & 2 & 1 & 4 & 3 & 10 & $41.7 \%$ \\
\hline Total & 12 & 12 & 12 & 12 & 6 & 6 & 6 & 6 & 24 & $100 \%$ \\
\hline
\end{tabular}

Al establecer una relación de mayor confianza, el 58.3\% de los entrevistados afirma emplear el voseo. No obstante, se mantiene el uso del ustedeo con un buen porcentaje de $41.7 \%$. Esto indica que, aunque los niveles de confianza aumenten gradualmente, no se exceden de forma repentina especialmente en lo concerniente a las féminas, puesto que la mayoría prefirió continuar utilizando el ustedeo como manifestación de distanciamiento, en cambio la mayoría de los entrevistados varones prefieren vosear en este contexto.

\section{Tabla 17.}

¿Cómo te dirigís a alguien cuando le estás pidiendo un favor?

\begin{tabular}{|c|c|c|c|c|c|c|c|c|c|c|}
\hline Variantes & \multicolumn{2}{|c|}{ Nivel generacional } & \multicolumn{2}{|c|}{ Nivel sexual } & \multicolumn{3}{|c|}{ Grupo individual } & total & \multirow{2}{*}{$\%$} \\
\cline { 2 - 10 } & Mayores & jóvenes & Hombres & Mujeres & $\mathrm{HM}$ & $\mathrm{HJ}$ & $\begin{array}{c}\mathrm{M} \\
\mathrm{M}\end{array}$ & $\mathrm{MJ}$ & & \\
\hline Vos & 2 & 3 & 4 & 1 & 2 & 2 & 0 & 1 & 5 & $20.8 \%$ \\
\hline Usted & 10 & 9 & 8 & 11 & 4 & 4 & 6 & 5 & 19 & $79.2 \%$ \\
\hline Total & 12 & 12 & 12 & 12 & 6 & 6 & 6 & 6 & 24 & $100 \%$ \\
\hline
\end{tabular}

Cuando se trata de pedir un favor, la forma que prevalece es el ustedeo (79.2\%), el voseo representa el $20.8 \%$ de uso. Esto se debe a que al manifestarle una necesidad personal a otro individuo se debe realizar con un alto grado de respeto y humildad, si se pretende alcanzar el objetivo deseado. Solo en cuanto al sexo se observaron diferencias, pues en las mujeres hay mayor consenso para tratar de usted en esta situación. 


\section{Tabla 18.}

¿Cómo te dirigís a una persona cuando estás enojado?

\begin{tabular}{|c|c|c|c|c|c|c|c|c|c|c|}
\hline Variantes & \multicolumn{2}{|c|}{ Nivel generacional } & \multicolumn{2}{|c|}{ Nivel sexual } & \multicolumn{3}{|c|}{ Grupo individual } & total & \multirow{2}{*}{$\%$} \\
\cline { 2 - 10 } & Mayores & Jóvenes & Hombres & Mujeres & $\mathrm{HM}$ & $\mathrm{HJ}$ & $\begin{array}{c}\mathrm{M} \\
\mathrm{M}\end{array}$ & $\mathrm{MJ}$ & & \\
\hline Vos & 10 & 11 & 10 & 11 & 5 & 5 & 5 & 6 & 21 & $87.5 \%$ \\
\hline Usted & 2 & 1 & 2 & 1 & 1 & 1 & 1 & 0 & 3 & $12.5 \%$ \\
\hline Total & 12 & 12 & 12 & 12 & 6 & 6 & 6 & 6 & 24 & $100 \%$ \\
\hline
\end{tabular}

Los informantes afirmaron que las demostraciones de enojo son expresadas con la forma de tratamiento del voseo (87.5\%, lo cual demuestra otra connotación del voseo: hostilidad e indignación. El uso del ustedeo solo se presenta en un $12.5 \%$.

\section{CONCLUSIONES}

A través del estudio de las formas de tratamiento pronominales realizado en la comunidad José Benito Escobar del municipio de Mateare se logró dar respuesta a los objetivos planteados en el presente trabajo, por lo tanto, llegamos a las siguientes conclusiones:

Las formas de tratamiento utilizadas en la comunidad de estudio son únicamente el ustedeo y el voseo, dejando el uso del tuteo solo para el lenguaje escrito, o para relacionarse con personas que también lo utilizan, como los extranjeros.

Entre la mayoría de los informantes se notó el uso del ustedeo por respeto, en el que influye mucho la edad, pues si el receptor es mayor, será tratado de usted, pero si es de menor edad, recibirá el voseo como tratamiento. Otro factor determinante para el uso de dicha forma de tratamiento es el distanciamiento entre los interlocutores, por ejemplo, en la relación alumno - maestro y empleado - jefe. Cabe destacar que este resultado es corroborado por la totalidad de los informantes, quienes asocian directamente el pronombre usted con el respeto, según los resultados reflejados en las tablas número 11 y 14 del análisis.

Los factores predominantes para el uso del voseo son la confianza existente entre los interlocutores y el tipo de relación establecida, pues si son amigos, hermanos, novios o esposos la usanza es vosearse, pues se trata de una relación simétrica.

Por otra parte, aunque la mayoría de los entrevistados aseguran que no hacen diferencias en cuanto a posición social, se evidencia el uso del ustedeo hacia personas que representan un mayor cargo jerárquico como médicos, jefes y docentes, aunque también estas personas suelen dispensar usted en el trato con sus interlocuto- 
res, pero este tratamiento varía con personas de menor estrato social, como las asistentes del hogar o el chofer de un bus, en los que se presenta la alternancia vos $\sim$ usted.

Por otro lado, en cuanto a las relaciones de distanciamiento (un desconocido, el chófer del bus) es notoria la preponderancia de la variable edad, puesto que las personas mayores indicaron el uso del voseo; en el caso de los jóvenes, fue diferente, pues prefieren la forma de tratamiento de ustedeo para entablar este tipo de relación social con desconocidos.

Entre las situaciones pragmáticas más notables, se pueden mencionar el uso del vos cuando el hablante se dirige a una persona con la cual está enojado. Por el contrario, cuando se está pidiendo un favor predomina el uso de usted, debido a que generalmente el hablante se presenta con una actitud de cortesía, dándole mayor importancia a la persona a quien le está solicitando el favor, con la intención de persuadirla.

En cuanto a las actitudes lingüísticas, es de gran interés notar que los informantes prefieren ser tratados de usted, pronombre que también es considerado "correcto" por los encuestados, a pesar de que el vos es forma de tratamiento más frecuente en el habla cotidiana de los nicaragüenses.

\section{REFERENCIAS}

Alemán A., Picado J. y Talavera M., (2017). Las formas de tratamiento en los jóvenes del municipio de Estelí: perspectivas dialectal y sociolingüística. UNAN -Managua.

Castro, A. (2001). Los pronombres de tratamiento en el español de Honduras. Honduras: Lincom Europa.

Christiansen A. (2012). Creencias y actitudes lingüísticas acerca de las formas de tratamiento en Nicaragua. Universidad de Bergen, Noruega.

Díaz, Tania \& López, Christian. (2013). Morfosintaxis del español de Nicaragua en El español hablado en América Central: Nivel morfosintáctico. Madrid: Editorial Vervuert.

Mendoza (2011). El voseo en el español nicaragüense. UNAN Managua. Nicaragua. Recuperado de menfrancismoran.blogspot.com/2011/03.el-voseo-en-el-español-nicaraguense-html?m=1

Quezada P. (2013). Español hablado en América Central: nivel morfosintáctico. Madrid

Uribe, Ó. (1970) Sociolingüística: una introducción a su estudio. Universidad Nacional Autónoma de México. 